\title{
B Lymphoblastic Leukemia/Lymphoma, BCR-ABL1-Like
}

National Cancer Institute

\section{Source}

National Cancer Institute. B Lymphoblastic Leukemia/Lymphoma, BCR-ABL1-Like. NCI Thesaurus. Code C129787.

B lymphoblastic leukemia/lymphoma with translocations involving tyrosine kinases, or translocations involving either the cytokine receptor-like factor 2 (CRLF2) or rearrang ements leading to truncation and activation of the erythropoietin receptor (EPOR). It is associated with an adverse prognosis. 